\title{
The rise and rise of cerebral small vessel disease: implications for vascular cognitive impairment and dementia
}

\author{
Raj N Kalaria*,1(D), Yoshiki Hase ${ }^{1} \&$ Masafumi Ihara² \\ ${ }^{1}$ Neurovascular Research Group, Institute of Neuroscience, Newcastle University, Newcastle upon Tyne, UK \\ ${ }^{2}$ Department of Stroke \& Cerebrovascular Diseases, National Cerebral \& Cardiovascular Centre, Osaka, Japan \\ *Author for correspondence: Tel.: +44 0191208 1352; Fax: +44 0191208 1301; raj.kalaria@newcastle.ac.uk
}

First draft submitted: 16 February 2019; Accepted for publication: 22 February 2019; Published online: 4 June 2019

Keywords: aging $\bullet$ carotid artery disease $\bullet$ cerebral amyloid angiopathy $\bullet$ small vessel disease $\bullet$ vascular brain injury - white matter hyperintensity

\section{The significance of cerebral small vessel disease}

Heterogeneity of cerebrovascular disorders has been realized for eons but brain or cerebral small vessel disease (SVD) is now in vogue. SVD seems to be distinguished in all types of dementias. This is largely due to advances in neuroimaging given that the resolution of magnetic resonance imaging (MRI) appears to improve day by day. We can now detect a repertoire of SVD features once only visible with the simple microscope. The definition of SVD presupposes to sharpen even more with the advent of high tensile MR scanners. White matter hyperintensities (WMH) on T2-weighted MRI have been the focus of a raft of well-meaning studies. They are regarded as the radiological signature of SVD. Frequencies of WMH or white matter (WM) lesions range 11-21\% in 64 year olds and increase to $94 \%$ by age 82 , with slightly higher frequency in women. WMH are more common and extensive in patients with cardiovascular risk factors and increase risk of stroke, dementia and death [1]. The heritability of WMH is as high as $45-73 \%$. WMHs are also associated with a greater decline in global cognitive performance and many domains of executive function [2]. Diffusion tensor imaging indicates microstructural changes within WM fibers in the frontal lobe are correlated with performance in cognitive domains including executive function, working memory and processing speed. Furthermore, lesion symptom mapping data and information from probabilistic WM atlases suggest that the anterior thalamic radiation is the major anatomical structure involving processing speed. These findings provide support for the role of frontal-subcortical circuits in cerebral SVD and vascular cognitive impairment. However, these tract changes are also apparent in variants of frontotemporal dementias. The current radiological definition of SVD includes lacunar infarcts, perivascular spacing, focal atrophy, microbleeds and microinfarcts [3]. These SVD features are invariably described to be present in different dementias even if thought to result from parallel processes with no interactions between vascular and neurodegenerative changes [4].

WMH of vascular origin are common in late onset neurodegenerative dementias including Alzheimer's disease (AD) $[2,5]$. Their presence in neurodegenerative diseases could reflect pathological processes other than those involved in SVD, in other words, that nonvascular damage could increase fluid motion in discrete areas of the WM to result in hyperintense signal [6]. However, it is not unlikely that there is a shared mechanism incorporating both vascular brain injury and neurodegenerative changes in $\mathrm{AD}$. Remarkably, recent findings from the Dominantly Inherited Alzheimer Network (DIAN) indicated that mutation carriers had greater total WMH volumes, which appeared to increase approximately 6 years prior to expected symptom onset [7]. Given the propensity for a posterior distribution of WMH, cerebral amyloid angiopathy (CAA), which tends to be distributed in posterior brain regions is thought to be one mediating factor. These findings suggest WMHs are a core feature of AD and a potential therapeutic target. There can be no doubt that WMH should be integrated into pathogenic models of the disease [7].

Pathological correlates of WMH when SVD is obvious suggest demyelination, axonal abnormalities, clasmatodendrosis, microglial activation, hemosiderin deposits, arteriolosclerosis and blood-brain barrier (BBB) dysfunction secondary to degrees of vascular brain injury [8]. Vessel changes in the cerebral and the systemic vasculature related

Future Medicine 
to WMH and other features of SVD likely begin during a considerable period prior to the manifestation of an overt stroke-like event or cerebrovascular accident. One manner in which this is evident is silent infarcts. Over 20\% of healthy elderly exhibit clinically silent MRI-defined silent brain infarcts and up to $50 \%$ of these are detected in those over 60 years [9]. While subtle deficits in physical and cognitive function associated with these changes commonly go unnoticed in older age, they increase risk of vascular cognitive impairment and dementia. Aging-related vascular degeneration involves sclerosis of cerebral brain vessels within and at the surfaces including the perforating and medullary arteries and arterioles, capillaries and venules. Systematic evaluation of the natural history and staging of SVD pathology indicated that vessel wall modifications such as arteriolosclerosis or deposition of amyloid-like proteins were the most common and earliest changes [10]. These were followed by perivascular spacing with lacunar and regional microinfarcts occurring as consequent but independent processes. The regional progression of the changes were from neocortical to subcortical structures. Clearly, SVD in early stages and during disease progression configures vascular dementia with the highest burden of SVD pathology but SVD no doubt influences the pathological phenotype of $\mathrm{AD}$ and other disorders including dementia with Lewy bodies, and probably the whole dementia spectrum expressing greater burden of SVD pathology than aging controls [10].

Besides age, hypertension and diabetes mellitus are the main risk factors for SVD. Whereas we do not fully understand how diabetes might lead to SVD [11], the deleterious effects of increased blood pressure are mediated by structural changes in smaller arteries leading to arteriolosclerosis. Chronic hypertension during aging can have two main consequences. One, it aggravates atherosclerotic changes in both extracranial and intracranial larger arteries, promoting to extend more distally into the intracranial compartment of smaller arteries and arterioles. The leptomeningeal arteries over the convexities in hypertensives harden appearing-like but not quite as larger atherosclerotic vessels. The progressive segmental loss of myocytes with replacement by collagen fibers promotes loss of elasticity to dilate and stiffens to constrict in response to variations of systemic blood pressure or autoregulation, which in turn causes fluctuations in blood flow response and tissue perfusion. Two, the persistent high pressure leads to focal disruption of capillaries, particularly in the deeper structures. SVD pathology likely leads to edema and damage of the BBB with chronic leakage of fluid and macromolecules in the WM. The consequences of these changes may be multiple lacunar infarcts and incidental microinfarcts occurring in both the deep gray matter and WM. Miliary micro-aneurysms arise in the context of hypertension, at weakened sites in vessel walls. The walls of the aneurysms are composed of hyaline connective tissue, damaged myocytes and elastica interna that may rupture to produce globular haemorrhages. They are transformed into fibrocollagenous balls, evident as complex tortuosities, when they heal due to thrombosis and fibrosis. They are most common at the interface between the gray matter and WM almost in all common dementias microvessel changes are remarkably common to all neurodegenerative diseases and the WM is particularly vulnerable irrespective of primary ischemic disease or a proteinopathy [12].

Typical SVD may also exhibit small vessel atherosclerosis or microatheroma found in proximal segments of penetrating arteries at junctions of branching and parent arteries and in parent vessels overlying the branch origin. The pathogenesis of atherosclerosis in small cerebral vessels does not differ substantially from that in extracranial vessels but are characterized by macrophages and nearly complete stenosis [13]. Likely extending from the repercussions of the systemic vascular system, the breach of the BBB occurs focally in selective areas of the aging brain. Using advanced dynamic contrast-enhanced MRI sequences with high spatial and temporal resolutions, BBB breakdown in the hippocampus was found to be an early event. The breach was worse in individuals with mild cognitive impairment as prodromal to $\mathrm{AD}$ [14]. Consistent with this, multifactorial data-driven analysis from the Alzheimer's Disease Neuroimaging Initiative suggested that intrabrain vascular dysregulation is an early pathological event during disease development and high abnormality levels are also observed for specific proteins associated with the function of the microvasculature [15].

The radiological signature of CAA includes posterior dominant WMH, lobar microbleeds, dilated perivascular spaces and multiple areas of superficial siderosis. While different amyloid proteins can cause intracranial CAA, the most common is amyloid- $\beta$ angiopathy, which is frequent in AD. SVD pathology in CAA is characterized by progressive segmental arteriolosclerosis involving the medial-adventitial layers of intracranial arteries. Of the two types of CAA, Type I is associated with capillaries implicating focal BBB damage. CAA is more common in individuals with infarction and hemorrhages and an independent substrate for cognitive impairment. DIAN studies suggest CAA is related to WM changes in familial AD [16]. Mutation carriers were more likely to have cerebral microbleeds than noncarriers and patients with microbleeds had higher WMH volumes. Although there was some 
co-dependency between WMH and microbleeds, these observations highlight that WMH represents a core feature of $\mathrm{AD}$ independent of vascular forms of amyloid- $\beta$ [16].

Sporadic SVD is by no means a pure disease like CADASIL or cerebral autosomal dominant arteriopathy with subcortical infarcts and leukoencephalopathy. It almost definitely coexists with degrees of atherosclerosis involving large extracranial vessels and cardioembolic disease [17]. Indeed, carotid artery stenosis is associated with markers of cerebral SVD such as WM changes, lacunar and nonlacunar infarcts. Elderly stroke survivors with typical SVD features and severe carotid artery disease developed greater numbers of cortical rather than subcortical infarcts and that severe disease was not necessarily associated with large infarcts. Microemboli from the cardiovascular system [18] could also be responsible for the numerous small infarcts or microinfarcts evident in patients who develop dementia. The hype of SVD might be dampened if data from carotid intima-media thickness measures, used as a noninvasive ultrasound marker of early atherosclerosis, were better known. Aging-associated central arterial stiffness may also increase SVD features in the elderly. Recent observations showed changes in heart-carotid pulse wave velocity were associated not only with greater volumes of WMH but confirmed there is an association between systemic vascular disease and amyloid- $\beta$ deposition in individuals without dementia [19]. As technological advances occur in imaging, we are likely to uncover more SVD and its various rebirths across the dementias. Still it is timely to implement therapeutic and preventative measures [20] to improve functional properties of the small vessels of the brain in the context of aging.

\section{Author's contributions}

All authors contributed to critically revising the manuscript for important intellectual content, and approved the final version of the manuscript for submission.

\section{Financial \& competing interests disclosure}

Our work is supported by previous grants from the Alzheimer's Research, UK (ARUK PG2013-22) and Medical Research Council, UK (MRC, G0500247). Y Hase was supported by SENSHIN Medical Research Foundation, Osaka, Japan and The Great Britain Sasakawa Foundation, London, UK. The authors have no other relevant affiliations or financial involvement with any organization or entity with a financial interest in or financial conflict with the subject matter or materials discussed in the manuscript. This includes employment, consultancies, honoraria, stock ownership or options, expert testimony, grants or patents received or pending, or royalties.

No writing assistance was utilized in the production of this manuscript.

\section{Open access}

This work is licensed under the Creative Commons Attribution-NonCommercial-NoDerivatives 4.0 Unported License. To view a copy of this license, visit: http://creativecommons.org/licenses/by-nc-nd/4.0/

\section{References}

1. Debette S, Markus HS. The clinical importance of white matter hyperintensities on brain magnetic resonance imaging: systematic review and meta-analysis. BMJ 341, c3666 (2010).

2. Prins ND, Scheltens P. White matter hyperintensities, cognitive impairment and dementia: an update. Nat. Rev. Neurol. 11(3), 157-165 (2015).

3. Wardlaw JM, Smith C, Dichgans M. Mechanisms of sporadic cerebral small vessel disease: insights from neuroimaging. Lancet Neurol. 12(5), 483-497 (2013).

4. Toledo JB, Arnold SE, Raible K et al. Contribution of cerebrovascular disease in autopsy confirmed neurodegenerative disease cases in the National Alzheimer's Coordinating Centre. Brain 136(Pt 9), 2697-2706 (2013).

5. Frings L, Yew B, Flanagan E et al. Longitudinal grey and white matter changes in frontotemporal dementia and Alzheimer's disease. Plos ONE 9(3), e90814 (2014).

6. Erten-Lyons D, Woltjer R, Kaye J et al. Neuropathologic basis of white matter hyperintensity accumulation with advanced age. Neurology 81(11), 977-983 (2013).

7. Lee S, Viqar F, Zimmerman ME et al. White matter hyperintensities are a core feature of Alzheimer's disease: evidence from the dominantly inherited Alzheimer network. Ann. Neurol. 79(6), 929-939 (2016).

8. Chen AQ, Akinyemi RO, Hase $\mathrm{Y}$ et al. Frontal white matter hyperintensities, clasmatodendrosis and gliovascular abnormalities in aging and post-stroke dementia. Brain 139, 242-258 (2016).

9. Vermeer SE, Longstreth WT, Jr, Koudstaal PJ. Silent brain infarcts: a systematic review. Lancet Neurol. 6(7), 611-619 (2007). 
10. Deramecourt V, Slade JY, Oakley AE et al. Staging and natural history of cerebrovascular pathology in dementia. Neurology 78(14), 1043-1050 (2012).

11. Bello-Chavolla OY, Antonio-Villa NE, Vargas-Vazquez A, Avila-Funes JA, Aguilar-Salinas CA. Pathophysiological mechanisms linking type 2 diabetes and dementia: Review of evidence from clinical, translational and epidemiological research. Curr. Diabetes Rev. doi:10.2174/1573399815666190129155654 (2019) (Epub ahead of print).

12. Hase Y, Ding R, Harrison G et al. White matter capillaries in vascular and neurodegenerative dementias. Acta Neuropathol. Commun. 7(1), 16 (2019).

13. Kalaria RN, Perry RH, O’brien J, Jaros E. Atheromatous disease in small intracerebral vessels, microinfarcts and dementia. Neuropathol. Appl. Neurobiol. 38(5), 505-508 (2012).

14. Montagne A, Barnes SR, Sweeney MD et al. Blood-brain barrier breakdown in the aging human hippocampus. Neuron 85(2), 296-302 (2015).

15. Iturria-Medina Y, Sotero RC, Toussaint PJ, Mateos-Perez JM, Evans AC; Alzheimer's Disease Neuroimaging I. Early role of vascular dysregulation on late-onset Alzheimer's disease based on multifactorial data-driven analysis. Nat. Commun. 7, 11934-11948 (2016).

16. Lee S, Zimmerman ME, Narkhede A et al. White matter hyperintensities and the mediating role of cerebral amyloid angiopathy in dominantly-inherited Alzheimer's disease. PLoS ONE 13(5), e0195838 (2018).

17. Caplan LR. Lacunar infarction and small vessel disease: pathology and pathophysiology. J. Stroke 17(1), 2-6 (2015).

18. Del Bene A, Makin SD, Doubal FN, Inzitari D, Wardlaw JM. Variation in risk factors for recent small subcortical infarcts with infarct size, shape, and location. Stroke 44(11), 3000-3006 (2013).

19. Hughes TM, Wagenknecht LE, Craft S et al. Arterial stiffness and dementia pathology: Atherosclerosis Risk in Communities (ARIC)-PET Study. Neurology 90(14), e1248-e1256 (2018).

20. Ihara M, Yamamoto Y. Emerging evidence for pathogenesis of sporadic cerebral small vessel disease. Stroke 47(2), 554-560 (2016). 